\title{
CDISC CDASH Terminology
}

National Cancer Institute

\section{Source}

National Cancer Institute. CDISC CDASH Terminology. NCI Thesaurus. Code C77527.

The terminology subset that includes terms relevant to the Clinical Data Interchange

Standards Consortium (CDISC) Clinical Data Acquisition Standards Harmonization (CDASH) group. 